\title{
ON LARGE DEVIATION PROBLEMS FOR SUMS OF RANDOM VARIABLES WHICH ARE NOT ATTRACTED TO THE NORMAL LAW
}

\author{
By C. C. Heyde \\ University of Sheffield
}

1. Introduction. Let $X_{i}, i=1,2,3, \cdots$, be a sequence of independent and identically distributed random variables with law $\mathfrak{L}(X)$, and write $S_{n}=\sum_{i=1}^{n} X_{i}$. Let $x_{n}, n=1,2,3, \cdots$, be a monotone sequence of positive numbers with $x_{n} \rightarrow \infty$ as $n \rightarrow \infty$ such that $x_{n}^{-1} S_{n} \rightarrow_{P} 0$. ("P" stands for convergence in probability). We shall call the probability $\operatorname{Pr}\left(\left|S_{n}\right|>x_{n}\right)$, or either of its component tail probabilities, a large deviation probability. This constitutes an extension of the ordinary concept of large deviation probabilities where the $X_{i}$ belong to some domain of attraction, $Z_{n}=B_{n}{ }^{-1} S_{n}-A_{n}$ are the normed and centered sums, and the probabilities $\operatorname{Pr}\left(\left|Z_{n}\right|>x_{n}\right)$ are called large deviation ones for $x_{n} \rightarrow \infty$ as $n \rightarrow \infty$.

In this paper, we shall consider random variables which do not belong to the domain of partial attraction of the normal distribution. That is, those for which (Lévy [4], 113)

$$
\lim \inf _{u \rightarrow \infty}\left[u^{2} \operatorname{Pr}(|X|>u) / \int_{|x| \leqq u} x^{2} d F(x)\right]>0,
$$

where $F(x)=\operatorname{Pr}(X \leqq x)$. We shall, in addition, make the restriction that for $\alpha>1$ and $\left\{x_{n}\right\}$ such that $x_{n}{ }^{-1} S_{n} \rightarrow_{p} 0$ as $n \rightarrow \infty$,

$$
0<\liminf _{n \rightarrow \infty}\left[\operatorname{Pr}\left(|X|>\alpha x_{n}\right) / \operatorname{Pr}\left(|X|>x_{n}\right)\right]
$$

The condition (2) is imposed to remove from consideration some laws whose distribution functions have widely spaced points of increase and for which the large deviation behaviour would be somewhat pathological.

Under conditions (1) and (2), we shall obtain a general expression for the order of magnitude of the large deviation probability in the form of (3) and (4) below. This provides a generalization of the work of Heyde [3] where the corresponding result (one sided version) was obtained for the case where the $X_{i}$ belong to the domain of attraction of a non-normal stable law. Apart from [3], there appears to be no other work directly on large deviation problems outside the domain of attraction of the normal distribution.

It is worth noting that the condition (2) goes some way towards implying condition (1). In this connection, we mention the following result which is a minor modification of a lemma of Doeblin [1]: If $\alpha>1$ and for $u>u_{0}$,

$$
\operatorname{Pr}(|X|>\alpha u) / \operatorname{Pr}(|X|>u) \geqq c_{\alpha}>0,
$$

where $\alpha^{2} c_{\alpha}>1$, then

$$
\lim \inf _{u \rightarrow \infty}\left[u^{2} \operatorname{Pr}(|X|>u) / \int_{|x| \leqq u} x^{2} d F(x) \geqq c_{\alpha}\left(1-1 / c_{\alpha} \alpha^{2}\right)>0 .\right.
$$

Received 13 February 1967. 


\section{Results.}

LEMma. If $\left\{x_{n}\right\}$ is a monotone sequence of positive numbess with $x_{n} \rightarrow \infty$ as $n \rightarrow \infty$, then $x_{n}{ }^{-1} S_{n} \rightarrow_{P} 0$ if and only if for every $\epsilon>0$,

(i) $n \operatorname{Pr}\left(|X|>\epsilon x_{n}\right) \rightarrow 0$ as $n \rightarrow \infty$,

(ii) $n x_{n}{ }^{-2} \int_{|x| \leqq x_{n}} x^{2} d F(x) \rightarrow 0$ as $n \rightarrow \infty$,

(iii) $n x_{n}{ }^{-1} \int_{|x| \leqq x_{n}} x d F(x) \rightarrow 0$ as $n \rightarrow \infty$.

This result is stated essentially for the sake of completeness. It follows immediately from the degenerate convergence criterion (Loève [5], 317). We note that when the $X_{i}$ do not belong to the domain of partial attraction of the normal distribution, condition (ii) is satisfied as a consequence of conditions (1) and (i).

Theorem. Suppose that $X_{i}, i=1,2,3, \cdots$, is a sequence of independent and identically distributed random variables satisfying the conditions (1) and (2). Further, $\left\{x_{n}\right\}$ is a monotone sequence of positive numbers such that $x_{n} \rightarrow \infty$ as $n \rightarrow \infty$ and $x_{n}{ }^{-1} S_{n} \rightarrow_{P} 0$. Then,

$$
\begin{aligned}
0<\liminf _{n \rightarrow \infty}\left[\operatorname{Pr}\left(\left|S_{n}\right|>x_{n}\right) / n \operatorname{Pr}\left(|X|>x_{n}\right)\right] \\
\quad \leqq \lim \sup _{n \rightarrow \infty}\left[\operatorname{Pr}\left(\left|S_{n}\right|>x_{n}\right) / n \operatorname{Pr}\left(|X|>x_{n}\right)\right]<\infty,
\end{aligned}
$$

or equivalently,

$$
\begin{aligned}
0<\liminf _{n \rightarrow \infty} & {\left[\operatorname{Pr}\left(\left|S_{n}\right|>x_{n}\right) / \operatorname{Pr}\left(\max _{k} \leqq n\left|X_{k}\right|>x_{n}\right)\right] } \\
& \leqq \lim \sup _{n \rightarrow \infty}\left[\operatorname{Pr}\left(\left|S_{n}\right|>x_{n}\right) / \operatorname{Pr}\left(\max _{k}\left|X_{k}\right|>x_{n}\right)\right]<\infty
\end{aligned}
$$

Proof. Take $\epsilon>0$ and denote by $A_{i}$ and $B_{i}$ the events $\left\{\left|X_{i}\right|>(1+\epsilon) x_{n}\right\}$ and $\left\{\left|\sum_{j=1, j \neq i}^{n} X_{j}\right|<\epsilon x_{n}\right\}$ respectively, $i=1,2, \cdots, n$. If $\bar{E}$ is the complement of $E$, we then have

$$
\begin{aligned}
\operatorname{Pr}\left(\left|S_{n}\right|>x_{n}\right) & \geqq \operatorname{Pr}\left[\bigcup_{i=1}^{n}\left(A_{i} \cap B_{i}\right)\right] \\
& \left.=\sum_{i=1}^{n} \operatorname{Pr}\left[\bigcap_{j=1}^{i-1} \overline{\left(A_{j} \cap B_{j}\right.}\right) \cap\left(A_{i} \cap B_{i}\right)\right] \\
& \geqq \sum_{i=1}^{n} \operatorname{Pr}\left[\bigcap_{j=1}^{i-1} \overline{A_{j}} \cap\left(A_{i} \cap B_{i}\right)\right] \\
& \geqq \sum_{i=1}^{n}\left\{\operatorname{Pr}\left(A_{i} \cap B_{i}\right)-\operatorname{Pr}\left(\mathrm{U}_{j=1}^{i-1} A_{j} \cap A_{i}\right)\right\} \\
& \geqq \sum_{i=1}^{n} \operatorname{Pr}\left(A_{i}\right)\left[\operatorname{Pr}\left(B_{i}\right)-(i-1) \operatorname{Pr}\left(A_{i}\right)\right] \\
& \geqq n \operatorname{Pr}\left(A_{1}\right)\left[\operatorname{Pr}\left(B_{1}\right)-n \operatorname{Pr}\left(A_{1}\right)\right] .
\end{aligned}
$$

Now $x_{n}{ }^{-1} S_{n} \rightarrow_{P} 0$ so $\operatorname{Pr}\left(B_{i}\right) \rightarrow 1$ as $n \rightarrow \infty$ and, given $\delta>0$ with $1-2 \delta>0$, we can choose $N_{1}$ so large that $\operatorname{Pr}\left(B_{i}\right)>1-\delta$ for $n \geqq N_{1}$. Further, from the lemma we see that $n \operatorname{Pr}\left(A_{i}\right) \rightarrow 0$ as $n \rightarrow \infty$, so that we can choose $N_{2}$ so large that $n \operatorname{Pr}\left(A_{i}\right)<\delta$ for $n \geqq N_{2}$. Thus, for $n \geqq N=\max \left(N_{1}, N_{2}\right)$, we obtain from $(5)$,

$$
\operatorname{Pr}\left(\left|S_{n}\right|>x_{n}\right) \geqq n(1-2 \delta) \operatorname{Pr}\left(|X|>(1+\epsilon) x_{n}\right)
$$

so that

$\operatorname{Pr}\left(\left|S_{n}\right|>x_{n}\right) / n \operatorname{Pr}\left(|X|>x_{n}\right) \geqq(1-2 \delta) \operatorname{Pr}\left(|X|>(1+\epsilon) x_{n}\right) / \operatorname{Pr}\left(|X|>x_{n}\right)$ 
and

$\liminf _{n \rightarrow \infty}\left[\operatorname{Pr}\left(\left|S_{n}\right|>x_{n}\right) / n \operatorname{Pr}\left(|X|>x_{n}\right)\right]$

$$
\geqq(1-2 \delta) \liminf _{n \rightarrow \infty}\left[\operatorname{Pr}\left(|X|>(1+\epsilon) x_{n}\right) / \operatorname{Pr}\left(|X|>x_{n}\right)\right]>0
$$

in view of condition (2).

In order to complete the proof we shall work in terms of the symmetrized random variables $X_{i}^{s}, i=1,2,3, \cdots$, making continued use of the weak symmetrization inequalities (Loève, [5], 245) to transfer the results.

Define

$$
\begin{aligned}
& X_{k n}^{s}=X_{k}^{s} \quad \text { if } \quad\left|X_{k}^{s}\right| \leqq 2 x_{n} \\
& =0 \text { otherwise, }
\end{aligned}
$$

and write $S_{n}^{s}=\sum_{k=1}^{n} X_{k}^{s}, S_{n n}^{s}=\sum_{k=1}^{n} X_{k n}^{s}$. We have

$$
\operatorname{Pr}\left(\left|S_{n}^{s}\right|>2 x_{n}\right) \leqq n \operatorname{Pr}\left(\left|X^{s}\right|>2 x_{n}\right)+\operatorname{Pr}\left(\left|S_{n n}^{s}\right|>2 x_{n}\right)
$$

and, using the weak symmetrization inequalities,

$$
\operatorname{Pr}\left(\left|X^{s}\right|>2 x_{n}\right) / \operatorname{Pr}\left(|X|>x_{n}\right) \leqq 2 \operatorname{Pr}\left(|X|>x_{n}\right) / \operatorname{Pr}\left(|X|>x_{n}\right)=2 .
$$

We now turn our attention to $\operatorname{Pr}\left(\left|S_{n n}^{s}\right|>2 x_{n}\right)$. From Chebyshev's inequality, we obtain

$$
\begin{aligned}
\operatorname{Pr}\left(\left|S_{n n}^{s}\right|>2 x_{n}\right) & \leqq\left(2 x_{n}\right)^{-2} E\left(S_{n n}^{s}\right)^{2} \\
& =n\left(2 x_{n}\right)^{-2} \int_{|x| \leqq 2 x_{n}} x^{2} d \operatorname{Pr}\left(X^{s} \leqq x\right),
\end{aligned}
$$

so that

$$
\begin{aligned}
& {\left[\operatorname{Pr}\left(\left|S_{n n}^{s}\right|>2 x_{n}\right) / n \operatorname{Pr}\left(|X|>x_{n}\right)\right]} \\
& \quad=\left[\operatorname{Pr}\left(\left|S_{n n}^{s}\right|>2 x_{n}\right) / n \operatorname{Pr}\left(\left|X^{s}\right|>2 x_{n}\right)\right]\left[\operatorname{Pr}\left(\left|X^{s}\right|>2 x_{n}\right) / \operatorname{Pr}\left(|X|>x_{n}\right)\right] \\
& \quad \leqq 2\left(2 x_{n}\right)^{-2} \int_{|x| \leqq 2 x_{n}} x^{2} d \operatorname{Pr}\left(X^{s} \leqq x\right) / \operatorname{Pr}\left(\left|X^{s}\right|>2 x_{n}\right),
\end{aligned}
$$

and lim $\sup _{n \rightarrow \infty}\left[\operatorname{Pr}\left(\left|S_{n n}^{s}\right|>2 x_{n}\right) / n \operatorname{Pr}\left(|X|>x_{n}\right)\right]<\infty$ since $X^{s}$ cannot belong to the domain of partial attraction of the normal distribution. We then have, from (7), lim $\sup _{n \rightarrow \infty}\left[\operatorname{Pr}\left(\left|S_{n}{ }^{s}\right|>2 x_{n}\right) / n \operatorname{Pr}\left(|X|>x_{n}\right)\right]<\infty$ and hence, using the weak symmetrization inequalities, $\lim \sup _{n \rightarrow \infty}\left[\operatorname{Pr}\left(\left|S_{n}-\operatorname{med} S_{n}\right|>2 x_{n}\right) /\right.$ $\left.n \operatorname{Pr}\left(|X|>x_{n}\right)\right]<\infty$. However, as $x_{n}{ }^{-1} S_{n} \rightarrow_{P} 0, x_{n}{ }^{-1}$ med $S_{n} \rightarrow 0$ as $n \rightarrow \infty$ and so, for $\epsilon>0$ and $n$ sufficiently large,

$$
\begin{aligned}
\operatorname{Pr} & \left(\left|S_{n}-\operatorname{med} S_{n}\right|>2 x_{n}\right) / n \operatorname{Pr}\left(|X|>x_{n}\right) \\
\geqq \operatorname{Pr}\left(\left|S_{n}\right|>(2+\epsilon) x_{n}\right) / n & \operatorname{Pr}\left(|X|>x_{n}\right) \\
=\left[\operatorname{Pr}\left(\left|S_{n}\right|>(2+\epsilon) x_{n}\right) / n\right. & \operatorname{Pr}\left(|X|>(2+\epsilon) x_{n}\right) \\
& \cdot\left[\operatorname{Pr}\left(|X|>(2+\epsilon) x_{n}\right) / \operatorname{Pr}\left(|X|>x_{n}\right)\right] .
\end{aligned}
$$

Using condition (2) and a simple transformation, it follows from (8) that 
$\lim \sup _{n \rightarrow \infty}\left[\operatorname{Pr}\left(\left|S_{n}\right|>x_{n}\right) / n \operatorname{Pr}\left(|X|>x_{n}\right)\right]<\infty$. This completes the proof of (3) and it just remains to establish the equivalence of (3) and (4). This, however, is easily deduced as, from Bonferroni's inequalities (Feller [2], 100), we have

$$
\begin{aligned}
n \operatorname{Pr}\left(|X|>x_{n}\right)\left[1-\frac{1}{2}(n-1) \operatorname{Pr}\right. & \left.\left(|X|>x_{n}\right)\right] \\
& \leqq \\
\operatorname{Pr}\left(\max _{k} \leqq n\right. & \left.\left|X_{k}\right|>x_{n}\right) \leqq n \operatorname{Pr}\left(|X|>x_{n}\right)
\end{aligned}
$$

and hence, in view of condition (i) of the lemma,

$$
\operatorname{Pr}\left(\max _{k \leqq n}\left|X_{k}\right|>x_{n}\right) \sim n \operatorname{Pr}\left(|X|>x_{n}\right) \text { as } n \rightarrow \infty .
$$

The required result follows and the proof of the theorem is complete.

\section{REFERENCES}

[1] Doeblin, W. (1940). Sur l'ensemble de puissances d'une loi de probabilité. Studia Math. $971-96$.

[2] Feller, W. (1957). An Introduction to Probability Theory and its Applications 1 (2nd edition). Wiley, New York.

[3] Heyde, C. C. (1967). A contribution to the theory of large deviations for sums of independent random variables. $Z$. Wahrscheinlichkeitstheorie verw. Gebiet. 7 303-308.

[4] Lévy, P. (1954). Théorie de l'addition des Variables Aléatoires (Deuxième édition). Gauthier-Villars, Paris.

[5] Lò̀ve, M. (1963). Probability Theory (3rd edition). Van Nostrand, New York. 